\title{
Aportaciones de la diacronía a la enseñanza de la morfología léxica en el aula*
}

\section{Contributions of diachrony to the teaching of lexical morphology in the Secondary Education}

\section{Cristina Buenafuentes de la Mata}

Universidad Autónoma de Barcelona

\section{Cristina.Buenafuentes@uab.cat}

ORCID ID: http://orcid.org/0000-0001-5931-1290

DOI: $10.17398 / 1988-8430.35 .1 .45$

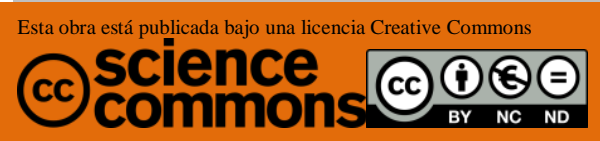

Fecha de recepción: 15/04/2021 Fecha de aceptación: 03/05/2021

Buenafuentes de la Mata, C. (2022). Aportaciones de la diacronía a la enseñanza de la morfología léxica en el aula. Tejuelo, 35(1), 45-70.

Doi: https://doi.org/10.17398/1988-8430.35.1.45

\footnotetext{
* Este trabajo se ha realizado en el marco de los Proyectos de Investigación financiados por el MICINN y FEDER FFI2017-87140-C4-1-P y PGC2018-094768-B-100 y por la CIRIT del Comissionat per Universitats $i$ Recerca de la Generalitat de Catalunya (2017 SGR 1251). Asimismo, se integra en las líneas de trabajo que promueve la asociación GrOC (Gramática Orientada a las Competencias: https://sites.google.com/view/groccast/inicio).
} 
Resumen: La morfología léxica es uno de los contenidos curriculares de la materia de Lengua y literatura castellana, ya que el conocimiento de la estructura interna de las palabras y sus procesos de formación son importantes para la mejora de la competencia comunicativa del alumnado. En este artículo se muestra la viabilidad de la incorporación de algunos conceptos y procesos de índole diacrónica en la enseñanza de la morfología léxica en Secundaria y Bachillerato. A pesar de asumir que, en toda la Enseñanza Media, el enfoque principal en el análisis de la estructura morfológica de las palabras complejas, de las relaciones entre sus constituyentes y de los procesos de formación de palabras debe ser el sincrónico, este trabajo pone de manifiesto que el empleo de la diacronía no solo contribuye a aumentar el alcance explicativo y descriptivo del plano sincrónico, sino que proporciona a los docentes argumentos sólidos para hacer reflexionar a los estudiantes sobre los hechos lingüísticos actuales que, al fin y al cabo, no dejan de ser el resultado de una evolución histórica.

Palabras clave: morfología; diacronía; gramática; competencia comunicativa; lengua española.
Abstract: Due to its importance in improving students' communicative competence, Lexical Morphology, i.e., the knowledge of the internal structure of words as well as their formation processes, is taken as part of the curriculum content of the subject "Spanish Language and Literature". In this paper, we discuss the viability of including certain diachronic concepts and processes in the teaching of Lexical Morphology during Secondary Education. Despite the assumption that the analysis of the morphological structure of complex words, as well as the relationships between their constituents and the word formation processes should have a synchronic major focus for the duration of Secondary Education, in this paper the application of diachrony will be proven not only useful for increasing the explanatory and descriptive power of synchronic means, but also capable of providing teachers with solid arguments enabling students to reflect on current linguistic phenomena, which, after all, are results of historical evolution.

Keywords: morphology; diachrony; grammar; communicative competency; Spanish. 


\section{$\int$ ntroducción}

Es una evidencia que la diacronía está ausente en la actualidad ${ }^{1}$ de la enseñanza de la morfología léxica en la Educación Secundaria y en el Bachillerato. Solo basta con abrir cualquier libro de texto de esos niveles para darse cuenta de que no se ofrecen informaciones de carácter histórico ni cuando se abordan los contenidos sobre gramática, en general, ni en el tratamiento de la morfología léxica, en particular. Por consiguiente, la enseñanza de la estructura interna de las palabras, así como de los procesos de lexicogénesis, aspectos cruciales en la enseñanza de la morfología según establecen los currículos educativos desde los cursos iniciales hasta el Bachillerato, se enfoca desde una perspectiva claramente sincrónica ${ }^{2}$. Esto puede ser debido, seguramente,

\footnotetext{
${ }^{1}$ Esto no siempre ha sido así, ya que en épocas pasadas la gramática histórica y la historia de la lengua se habían tenido en cuenta en los planes de estudio de la Enseñanza Media, tal y como pone de manifiesto Martín Zorraquino (2000, pp. 7677).

2 Nos basamos en los currículos de Educación Secundaria Obligatoria y de Bachillerato en el ámbito lingüístico (lenguas catalana y castellana) de la comunidad autónoma de Catalunya, disponibles en http://educacio.gencat.cat/ca/departament/publicacions/colleccions/curriculum/ curriculum-eso/ y $\quad \mathrm{http} / /$ educacio.gencat.cat/ca/departament/ publicacions/colleccions/curriculum/curriculum-batxillerat/. Ambos currículos vienen determinados por los Decretos 187/2015 y 142/2008, que regulan en esta comunidad
} 
a que se asume que, para poder dar cuenta de la estructura interna de una palabra o de los procesos que intervienen en su formación, no se precisa tener conocimientos de etimología ni de morfología histórica. Por ello y dado que muchas irregularidades en morfología solo son explicables a partir de una evolución histórica, no resulta extraño que la enseñanza de la morfología léxica en Secundaria y en Bachillerato se centre en la regularidad morfológica.

No es nuestro propósito en este artículo defender que la diacronía sea el enfoque principal de la enseñanza de la morfología en estos niveles, sino mostrar que en determinadas ocasiones su implementación puede resultar útil, porque permite interpretar mejor los hechos lingüísticos sincrónicos. Tal y como señala Lleal (2008), “el profesor ha de conocer las líneas generales de la evolución [...]. Los datos que nos proporciona la diacronía [...] nos permiten alcanzar explicaciones coherentes con la realidad de los hechos" (p. 151). Por tanto, partimos de la base de que, aunque se pueda abordar el análisis de la morfología léxica sin atender a su evolución, en realidad, "una lengua aun considerada en un aislamiento total y en un punto de su evolución contiene información más o menos implícita sobre estados anteriores" (Michelena, 1963, p. 17). Sirva como ejemplo la Nueva gramática de la lengua española de la RAE y ASALE (2009), obra de referencia para investigadores y docentes, que, de manera recurrente, hace uso de la diacronía en la descripción gramatical. En palabras de Echenique (2011), "al abrir la Nueva Gramática se advierte, de inmediato, que la historia de la lengua impregna la obra en todos los grandes apartados, [...]; el recurso a la diacronía es, en todo caso, una constante" (p. 162). De todos modos, no se trata de introducir más materia a los ya cuantiosos contenidos de lengua en la Enseñanza Media, sino de seleccionar aquella que nos va a resultar operativa desde el punto de vista de la práctica docente.

Teniendo en cuenta estas consideraciones, este trabajo tiene como objetivo fundamental mostrar la operatividad del empleo de determinados aspectos diacrónicos en la enseñanza de la morfología

la ordenación de las enseñanzas de la Educación Secundaria Obligatoria y del Bachillerato, respectivamente. 
léxica en Secundaria y en Bachillerato. De este modo, se va a demostrar que tender puentes entre la sincronía y la diacronía se convierte en una estrategia docente eficaz, porque no solo contribuye a comprender mejor los hechos lingüísticos actuales, sino también porque proporciona argumentos para su reflexión y para que, en definitiva, el aprendizaje de la morfología resulte significativo para el alumnado. Si bien la relevancia de la diacronía en la enseñanza de la lengua se ha puesto de manifiesto en varios trabajos centrados en la enseñanza del español como segunda lengua (Bravo Bosch, 1994; Díez del Corral, 2015; Fernández Martín, 2019), apenas se ha abordado esta cuestión en la enseñanza de la lengua en Secundaria y Bachillerato, de modo que este estudio quiere contribuir a llenar ese vacío.

Para acometer el objetivo de este trabajo, es necesario llevar a cabo un breve repaso de las cuestiones que se trabajan en Secundaria y Bachillerato en relación con la morfología léxica, pues ello nos va a proporcionar la base a partir de la cual introducir determinados aspectos diacrónicos. Este propósito se efectuará en el siguiente apartado.

\section{1.- Enseñanza de la morfología léxica: aspectos clave}

La enseñanza de la morfología léxica en Secundaria y en Bachillerato se centra en dos aspectos principalmente: en primer lugar, en el establecimiento de la estructura interna de la palabra y, por tanto, en la identificación de sus unidades de análisis (morfema, morfo, alomorfo, afijos, etc.) y, en segundo lugar, en la descripción de sus relaciones teniendo en cuenta reglas de formación de palabras (y, consecuentemente, en los mecanismos de formación como la derivación, la composición, etc.). Estos contenidos de índole más gramatical se vinculan estrechamente con la mejora de la competencia lingüística del alumnado que se persigue en toda la Enseñanza Media y que, por tanto, es una constante en todo el desarrollo curricular. El conocimiento de la estructura interna de las palabras y de sus reglas de formación permiten la decodificación (descomposición) de palabras existentes, lo que favorece la comprensión de producciones ajenas, pero también contribuye a la codificación (creación) de nuevas unidades, 
factor que potencia la creatividad lingüística. Por consiguiente, aunque en ocasiones los contenidos gramaticales se presenten como un conocimiento al margen de la competencia linguiística, la relación es evidente y hacerla visible en el aula está en manos del profesorado. Por ejemplo, una de las actuaciones que permiten evidenciar este hecho es realizar segmentaciones de palabras. La segmentación, tal y como señala Gil (2019), “es un proceso natural que realizan los hablantes intuitivamente" (p. 52) y, por consiguiente, partir de ese conocimiento previo que los alumnos poseen por defecto les muestra que la lengua les pertenece y que, como hablantes, poseen a priori argumentos para poder reflexionar sobre ella (Bosque, 2017).

Tomando como base las unidades de análisis y las reglas de formación de palabras, la morfología léxica analiza la estructura de las palabras teniendo en cuenta la siguiente premisa: una palabra tendrá estructura morfológica (se puede segmentar en unidades más pequeñas) si puede descomponerse a partir de reglas de formación de palabras sincrónicas, independientemente de su etimología. En este sentido, los hablantes codifican y descodifican palabras sin necesitar conocimientos etimológicos. Cualquier estudiante al que preguntemos por la estructura interna de tristeza, nos responderá que se trata de una palabra formada por el adjetivo triste y el sufijo de cualidad - eza, cuando, si acudimos a su etimología, esta palabra proviene del latín tristitia. No queremos entrar aquí en las diferencias evidentes entre el análisis morfológico sincrónico y el diacrónico (RAE y ASALE, 2009, § 1.6; SerranoDolader, 2012; Martín García y Varela, 2012), sino poner el foco de atención en esta diferencia, para asumir que, en Secundaria y en Bachillerato, hay que partir de esta premisa sincrónica en la enseñanza de la morfología léxica. Por tanto, desde esta perspectiva, tristeza tendrá estructura morfológica, pues se ignorará su etimología a la hora de segmentarla morfológicamente. La aplicación de este supuesto nos permitirá explicar la distinta estructura de determinadas palabras que, aparentemente, presentan una semejanza en cuanto a su formación. Por ejemplo, obsérvese el siguiente par mínimo ${ }^{3}$ :

${ }^{3}$ En este trabajo, se van a proponer ejemplos que siguen la metodología de los pares mínimos, pues consideramos que son un tipo de ejercicio que fomenta la reflexión. Para más información sobre estos ejercicios más competenciales, véase Bosque y 
a. La morbosidad a Juan le jugó una mala pasada

b. La curiosidad a Juan le jugó una mala pasada

Se puede comprobar que la diferencia entre las dos oraciones reside en los derivados morbosidad y curiosidad. Aparentemente, la determinación de su estructura morfológica podría ser análoga, pues ambas palabras parecen partir de una raíz a la que se le incorporan los sufijos -oso e -idad. Sin embargo, aunque este análisis es válido para el caso de morbosidad (morb(o) $\left.{ }^{4}+o s(0)+i d a d\right)$ no lo es para curiosidad, porque esta segmentación dejaría una raíz inexistente en español (curi(o)-, en este caso), y la existencia de la raíz es requisito imprescindible para la buena formación de una palabra en español. Por consiguiente, curiosidad presenta la siguiente estructura: curios(o)+idad. Este análisis se ha basado estrictamente en el supuesto antes presentado y, por tanto, ignora la etimología, ya que, curiosidad procede del latín curiositatis y, por tanto, desde la diacronía del español ${ }^{5}$, no posee estructura morfológica.

Asumir esta premisa claramente sincrónica en la enseñanza de la morfología en Secundaria y en Bachillerato lleva, por tanto, a plantearse la pregunta sobre qué lugar tiene entonces la diacronía en el análisis morfológico y si es operativo su empleo desde la perspectiva docente. Trataremos de responder a estas cuestiones a continuación.

Gallego (2016) y, para el caso particular de la morfología, Gil (2019) y Buenafuentes (2020).

${ }^{4}$ La marca de palabra o marca segmental, cuando es átona, se cancela al incorporar otro morfema. De ahí que se delimite con paréntesis.

${ }^{5}$ Sí que poseería estructura morfológica en la lengua latina, pues esta palabra se formó en latín mediante un proceso de sufijación. 


\section{2.- Aportaciones de la diacronía a la enseñanza de la morfología léxica sincrónica}

A pesar de la necesidad de partir de una perspectiva sincrónica en la enseñanza de la morfología, consideramos que introducir determinados aspectos relacionados con la diacronía en Secundaria y en Bachillerato puede ser útil desde el punto de vista de la práctica docente. De este modo, la diacronía puede ayudar a "regularizar" determinadas irregularidades de la morfología léxica del español. No queremos decir que tales irregularidades se conviertan en regulares, sino que la diacronía proporciona una explicación que da mayor alcance al análisis y descripción sincrónicos, pues, como se tratará de demostrar, permite determinar la estructura interna de un mayor número de palabras del español. Además, la perspectiva histórica proporciona argumentos para reflexionar sobre cómo el paso del tiempo deja huellas en la morfología y sobre que lo que se presentan como "irregularidades" no son producto del capricho o un hecho puntual de carácter excepcional, sino que pueden ser explicadas de manera coherente. Tal y como señalan Martín García y Varela (2012), "Hay irregularidades formales producto de la evolución [...] la perspectiva histórica nos va a permitir entender, desde la sincronía, las inconsistencias formales y/o semánticas que revelan" (p. 328). En los siguientes apartados, se ofrecen una serie de conceptos y procesos diacrónicos que pueden resultar operativos en la enseñanza de la morfología léxica sincrónica, pues permiten ampliar el alcance del análisis, explicar irregularidades y propiciar la reflexión sobre por qué los hechos linguiísticos son así y no son de otra manera. Con ello, en definitiva, favoreceremos que los estudiantes entiendan "la lengua en su conjunto y no como una serie de arbitrariedades y estructuras que hay que memorizar y aprender sin un porqué”. (Bravo Bosch, 1994, p. 391).

\section{1.- Conceptos diacrónicos operativos en la enseñanza de la morfología léxica sincrónica}

Uno de los aspectos ineludibles en la enseñanza de la morfología es el concepto de alomorfo (véase RAE y ASALE, 2019: s.v. alomorfo). Esta noción puede ver ampliado su valor explicativo si se adopta una 
perspectiva diacrónica, ya que la alomorfía puede venir determinada también por razones de tipo etimológico. Si limitamos la alomorfía solo a aquella condicionada fonológica o morfológicamente (por ejemplo, los alomorfos del prefijo in- o los que se relacionan con la formación del plural), habrá muchas palabras que van a escapar del análisis, porque no tendrán estructura morfológica. Consideramos, por consiguiente, que, dado que el concepto de alomorfía es básico en la enseñanza de la morfología, sería fácil introducir también el concepto de alomorfía diacrónica. Obsérvense las siguientes secuencias:

\section{a. Vestido dominguero}

b. Revista dominical

Las dos palabras derivadas de las dos secuencias son adjetivos de relación que tienen el mismo significado de 'perteneciente o relativo al domingo', aunque en su formación intervienen dos sufijos diferentes (-ero en la primera secuencia; - $a l$, en la segunda) ${ }^{6}$. La alomorfía en este caso se encuentra en la raíz: en la secuencia de a. esta es patrimonial (doming-), mientras que en la de b. es culta (dominic-). Si no adoptamos una perspectiva diacrónica en este caso de alomorfía, el derivado dominical no tendría estructura morfológica, pues, al aislar el sufijo -al, la raíz no existe en español (dominic(o)-). Otros ejemplos de este tipo serían norteño y septentrional, rural y campero, caprino y cabruno, doméstico y hogareño, entre otros. De este modo, si se asume que en español se dan casos de alomorfía diacrónica (en este caso, de raíces supletivas, véase RAE y ASALE, 2009), se podrán analizar todas aquellas palabras en las que se dé tal alternancia morfológica basada en el origen culto o patrimonial del morfema ${ }^{7}$. Por tanto, las raíces cultas inexistentes se justificarían dentro del análisis sincrónico si alternan con una forma patrimonial. Creemos que la diferencia entre culto y patrimonial puede ser reconocida de manera intuitiva por el alumnado (sin entrar en mayores detalles, solo a partir de la forma y yendo hacia el significado) y con más garantías si, además, están cursando la

\footnotetext{
'El comportamiento en este caso de los afijos y sus restricciones se tratarán más adelante.

7 Cabe advertir que no siempre se da esta alternancia. Determinados adjetivos de relación solo tienen una de las dos posibilidades y, en la mayoría de los casos, la base suele ser culta. Véanse los ejemplos (5) y (6).
} 
asignatura de latín, lo que muestra que introducir este tipo de contenidos puede hacer posible la transversalidad entre las diferentes lenguas que se trabajan en Secundaria y en Bachillerato.

Este concepto de alomorfía diacrónica permite también dar cuenta de las variantes alomórficas que presentan los afijos y que también se justifican diacrónicamente. Es el caso de los siguientes pares mínimos:

\section{a. Confesión forzosa}

b. Revelación forzosa

a. Hay que intercambiar los ingredientes

b. Hay que entremezclar los ingredientes

En estos ejemplos, se pone de manifiesto que algunos afijos con el mismo significado presentan variaciones alomórficas que responden a la distinción culto/patrimonial. Por ejemplo, el par mínimo de (3) da cuenta de las variantes -sión (culta) y -ción (patrimonial) para el sufijo nominalizador -ción 'acción y efecto de $\mathrm{X}$ ', mientras que en los ejemplos de (4) se observa el mismo fenómeno pero en el ámbito de la prefijación para entre- (patrimonial) e inter- (culto) con el valor de 'acción recíproca'. Como se desprende de estos casos, se trata de afijos productivos que presentan una variante culta que solo permite ser analizada en sincronía si se asume su origen histórico y su alternancia con un correlato patrimonial. En definitiva, consideramos que el concepto de alomorfía diacrónica contribuye a explicar mejor la estructura de determinadas palabras en las que la evolución ha dejado su huella. Al igual que no se enseña la prefijación o la sufijación memorizando todos los sufijos y prefijos del español, no se trataría de listar estas variantes alomórficas diacrónicas. En este sentido, los ejercicios de pares mínimos pueden resultar una estrategia docente idónea: permiten observar la diferencia, reflexionar sobre ella y establecer generalizaciones que puedan aplicarse a más situaciones semejantes.

Otro de los aspectos que se aborda en la enseñanza de la morfología en Secundaria y en Bachillerato son las reglas de formación 
de palabras (RFP) que son la base de los procesos de lexicogénesis (sufijación, prefijación, composición, principalmente). Las RFP se basan en la consideración de que la palabra presenta una estructura jerárquica en la que las unidades que la forman deben respetar una serie de restricciones de adjunción (Felíu, 2009, p. 57). Tales restricciones pueden ser de carácter fonológico (el prefijo re- no acepta bases que empiecen por vibrante), morfológico (el sufijo -mente rechaza bases previamente derivadas con el sufijo -ificar), categorial (el sufijo -ble rechaza bases cuya categoría gramatical no sea verbal) o semántico (el sufijo -ción solo acepta bases que expresen acción) y pueden ser impuestas por los afijos o por las bases. En este sentido, también las restricciones de las RFP pueden deberse a aspectos diacrónicos, de modo que los afijos o las bases pueden seleccionar los morfemas que se le adjuntan atendiendo a especificidades de carácter etimológico. Esto es lo que ponen de manifiesto los ejemplos que se exponen a continuación:

a. Leche corporal

b. Leche *cuerpal

a. Vaca lechera

b. Vaca *lactera

En los ejemplos de (5) y (6) se utilizan -al y -ero, $a$, sufijos que crean adjetivos de relación. A pesar de ser, por tanto, sufijos con el mismo valor semántico, poseen distintas restricciones a la hora de adjuntarse que tienen que ver con el origen etimológico de sus bases. Mientras -al exige que sus bases sean cultas (por este motivo, *cuerpal, con base patrimonial, no es válido), -ero, a no las admite, pues solo se incorpora a aquellas bases cuyo origen sea patrimonial. Abogamos por introducir también esta restricción de carácter diacrónico porque proporciona argumentos para poder explicar la (im)posibilidad de crear determinadas palabras y, con ello, se amplía el alcance de la descripción de los propios procesos de lexicogénesis.

Todos los aspectos diacrónicos que se han señalado hasta el momento son la evidencia de las huellas que ha dejado la lengua latina sobre el español y que son el producto de la evolución que experimenta 
cualquier sistema lingüístico. Sin embargo, el español también ha adoptado las RFP y los mecanismos de creación de nuevo léxico de las lenguas clásicas (griego y latín). Esto significa que el español toma de estas lenguas clásicas no solo el proceso de lexicogénesis sino también las unidades que se emplean para llevarlo a cabo. El ejemplo más claro de ello lo constituye la composición culta, es decir, el proceso morfológico que consiste en la unión de dos o más bases donde al menos una de ellas es un elemento culto (como en aeronave, hipódromo, cardiología o blefaroplastia). Se trata de un tipo de composición que, a pesar de su productividad y de su extensión en el español actual, no siempre se contempla en la enseñanza de la morfología en Secundaria y en Bachillerato a tenor de los contenidos de los libros de texto. Sin embargo, los hablantes conocen perfectamente este tipo de formaciones, pues, a pesar de crearse en el seno de lenguajes de especialidad, muchas de ellas se han extendido a la lengua general y eso ha propiciado que sean interpretables por parte de los hablantes no especializados (hipo $=$ 'caballo' + dromo $=$ 'carrera' $)$.

Como se desprende de los ejemplos, la composición culta no es más que la aplicación en español de la composición tal y como se realizaba en latín o en griego, lo cual deja un problema importante desde el punto de vista teórico: la inexistencia de los elementos que intervienen en la formación de esos compuestos cultos. Es decir, ni hipo- ni -dromo se encuentra entre las palabras del español, lo que imposibilita que estas voces compuestas sean segmentadas y, por tanto, quedarían fuera de las palabras complejas al no tener estructura morfológica. Sin embargo, es evidente que estos vocablos se crean por un proceso de formación de palabras, aunque este sea importado. De hecho, es posible que un elemento culto no existente se una a una palabra patrimonial para crear un vocablo nuevo (como en canódromo) o incluso que adquiera nuevos valores (como en botellódromo, donde dromo significa 'lugar' y no 'carrera' como en griego), lo cual indicaría que tienen funcionalidad como creadores de palabras en español, a pesar de que no existan como palabras autónomas en esta lengua. En este sentido, para solventar el problema teórico que plantean estos formantes desde el punto de vista de la descripción sincrónica de los procesos de formación de palabras, desde la perspectiva metodológica, 
resulta operativo introducir el concepto de tema de palabra inexistente (Iglesias, 2018), porque otorga a estos elementos cultos un estatuto morfológico (el de tema) y porque, a pesar de su inexistencia en español, permite que puedan ser descritos desde la sincronía como palabras complejas del español. Por consiguiente, utilizar en aula este concepto de corte diacrónico facilita la incorporación también de la composición culta como parte de los procesos de formación de palabras del español. Además, regulariza su naturaleza, ya que este tipo de composición queda asociada a la composición léxica (tocadiscos, sordomudo, pelirrojo ${ }^{8}$ ), con la única diferencia de que, en la primera, el proceso se lleva a cabo con piezas tomadas del griego o del latín que reciben el nombre de temas de palabras inexistentes.

La diacronía también permite dar cuenta de otras "irregularidades" en los procesos de formación de palabras. Si bien este tipo de cuestiones convendría reservarlas para cursos más avanzados, las comentamos aquí porque dan cuenta de las posibilidades que abre la incorporación de la diacronía a la enseñanza de la morfología. En este sentido, los compuestos léxicos suelen reflejar el orden de los constituyentes en la sintaxis, lo que implica que su núcleo (el elemento que impone los rasgos morfológicos y semánticos a todo el compuesto) se encuentra a la izquierda ${ }^{9}$. Así, en hierbabuena, el núcleo ${ }^{10}$ es el sustantivo hierba, porque impone sus rasgos morfológicos y semánticos a todo el compuesto (hierbabuena es un nombre femenino, como hierba, y además constituye su hipónimo, al ser un tipo de hierba). Sin embargo, un alumno avispado se dará cuenta de que no siempre los compuestos del español tienen el núcleo a la izquierda: vasodilatador, drogodependiente, catalanohablante o narcotraficante son compuestos que tienen el núcleo a la derecha. Lo que explica esta aparente

\footnotetext{
${ }^{8}$ De hecho, algunos compuestos léxicos tienen una vocal de enlace que imita la que se introducía entre los elementos de los compuestos latinos $(-i-)$ y griegos $(-o-)$, como se observa en pelirrojo y otros compuestos semejantes (perniquebrar, drogodependiente, agridulce, fofisano, entre otros).

${ }^{9}$ Las diferentes lenguas se comportan de forma diferente, lo cual puede dar pie a su contraste teniendo en cuenta la situación del núcleo dentro de la composición.

${ }^{10}$ Dado que el concepto de núcleo es motivo de debate en la teoría morfológica (véase Buenafuentes, 2021), convendría no profundizar demasiado en este aspecto y proporcionar ejemplos que no resulten problemáticos.
} 
irregularidad es que en realidad estos compuestos responden a lo que Martín García y Varela (2012) denominan la reactivación de un patrón morfo-histórico mediante el cual se crean compuestos en español según el orden de la composición derivada de la sintaxis latina, que situaba el núcleo a la derecha (del tipo armisonans, ignipotens, blandiloquens). Este tipo de contenidos deben implementarse necesariamente en cursos avanzados, en los que los alumnos puedan tener conocimientos previos de latín, que les permitan establecer el contraste entre la lengua española y su antecedente lingüístico y reconocer las consecuencias de esta relación genética e histórica en los procesos de formación de palabras. Tal y como señala Díez del Corral (2015) en su estudio aplicado a la enseñanza de lenguas extranjeras, pero que es totalmente extrapolable a la Enseñanza Media,

[...] acercarse a la historia del español proporciona al estudiante unas herramientas útiles para su comprensión y aprendizaje. El alumno adquiere una mayor conciencia de la lengua que está aprendiendo hasta el punto de que puede incluso influir en el aumento de su motivación [...]. (p. 224).

En definitiva, se ha demostrado que ciertos conceptos de corte diacrónico (alomorfía diacrónica, restricciones etimológicas de las RFP, tema de palabra inexistente) son operativos en la enseñanza de la morfología en Secundaria y en Bachillerato, pues permiten dar cuenta de la riqueza de los procesos de formación de palabras y de la huella que ha dejado la lengua latina en ellos, lo cual amplía el alcance descriptivo del análisis morfológico sincrónico.

\section{2.- Procesos diacrónicos operativos en la enseñanza de la morfología léxica sincrónica}

Otro de los contenidos recurrentes en la enseñanza de la morfología en Secundaria y en Bachillerato es el relacionado con los procesos de formación de palabras, como se ha abordado en el apartado precedente. Se trata de un aspecto ineludible en la morfología léxica, pero que carece de una secuenciación a la hora de ser incorporado en el aula, ya que se tiende a repetir lo mismo en todos los niveles de 
aprendizaje $\mathrm{e}^{11}$. Es obvio que la diferenciación de las palabras complejas que responden a un proceso prototípico de formación de palabras (sufijación, prefijación, composición) no reviste demasiada complejidad, por lo que puede ser explicada en niveles iniciales de la secundaria. Sin embargo, existen algunas palabras complejas que ponen de manifiesto los difusos límites entre los distintos mecanismos de lexicogénesis. Por ejemplo, ¿monodosis y pseudociencia son compuestos o palabras prefijadas? ¿Flequillo es una palabra compleja? ¿Responden al mismo proceso lexicogenésico las palabras televisión y telebasura o biología y biocombustible? ¿y botellódromo e hipódromo? Precisamente, en la delimitación de estas formaciones, puede resultar esencial tener en cuenta distintos aspectos de índole diacrónica.

Como se evidencia tanto en manuales de morfología como en los propios diccionarios, la nómina de prefijos y de sufijos suele variar según las fuentes. Esta vacilación en la clasificación tiene su origen, principalmente, en la caracterización de los elementos lexicogenésicos que son de origen culto (García Medall, 2019, p. 403). Si bien la caracterización de los morfemas que se sitúan delante de una base cuya naturaleza no es culta suele resultar sencilla (ante-, entre- o sobre-se considerarían, sin ninguna duda, prefijos), este hecho no resulta tan fácil cuando ese elemento es de origen culto, de modo que la clasificación de unidades como pseudo-, neo- o mono- y de las palabras en las que intervienen puede resultar más complicado. Tal y como señala la RAE y ASALE (2009: 668), atendiendo a la clase de palabras a las que se asimilan, los prefijos pueden ser adverbiales, preposicionales $\mathrm{y}$ adjetivales $^{12}$. Esta cuestión, si bien es independiente de que el prefijo sea de origen culto o patrimonial, tiene evidentes consecuencias para las unidades cultas que plantean dificultades de clasificación como los casos de neo-, pseudo- o mono-: cualquier elemento que se asocie a una de esas categorías se debe considerar un prefijo. Por tanto, pseudo'falso', neo- 'nuevo' o mono- 'uno', elementos que se asocian

\footnotetext{
${ }^{11}$ Esta falta de secuenciación se observa también en otros contenidos gramaticales relacionados con la morfología, como, por ejemplo, en la enseñanza de las categorías gramaticales (Buenafuentes y Fernández Serrano, en prensa).

${ }_{12}$ De todos modos, cabe señalar que no todas las preposiciones, adverbios o adjetivos tienen la posibilidad de funcionar también como prefijos.
} 
claramente a adjetivos, son prefijos cultos. En contraste, otros elementos cultos como aero- 'aire', bio- 'vida, cardio- 'corazón', que se asocian a nombres, no lo serían (constituirían lo que hemos denominado temas de palabras inexistentes). Resulta esencial introducir esta distinción en la enseñanza de la morfología léxica, porque permite distinguir las palabras resultado de la prefijación culta (neoconservador, pseudociencia, monodosis) de las que se han formado por composición culta (aeroespacial, cardiología, biocombustible), sin necesidad de memorizar una lista de prefijos ni de temas compositivos, ya que, a partir de su significado y de su adscripción categorial, se puede reconocer si se trata o no de prefijos cultos ${ }^{13}$.

Hasta aquí solo se ha tomado en consideración la prefijación. En el caso de la sufijación, mayoritariamente, los elementos cultos que se emplean en la formación de palabras se pueden asociar con sustantivos o adjetivos (que pueden ser derivados de un verbo latino o griego). Por ejemplo, -filia es un sustantivo que significa 'simpatía' o -cida un deverbal que significa 'exterminador'. Cabría clasificar estas unidades como temas cultos, al poder asociarse, categorial y semánticamente, a los sustantivos o adjetivos ${ }^{14}$. Además, otro de los rasgos que solo pueden tener los temas cultos (no los afijos) es la posibilidad de aparecer en primera o segunda posición de un determinado vocablo complejo (si bien no todos los temas cultos poseen esta característica). Por ejemplo, el elemento culto ptero-/-ptero 'ala' puede aparecer en primer lugar (pterodáctilo) o en segundo (chupóptero), comportamiento

${ }_{13}$ Seguramente se encontrarían ejemplos que escaparan a esta generalización, pero debemos centrar la enseñanza de la gramática en aquellos aspectos que permitan describir la mayoría de los casos y prescindir de aquellos que manifiesten un comportamiento aislado y particular.

${ }^{14}$ Esta caracterización no resulta tan sencilla, ya que, a pesar de que son asimilables al mismo tipo categorial, sustantivo o adjetivo en este caso, no todos los elementos cultos que se sitúan en segunda posición se clasifican como temas cultos: por ejemplo, -itis, que significa 'inflamación', se considera mayoritariamente un sufijo (hepatitis, faringitis en el DLE (véase el RAE y ASALE, 2014). Sin embargo, dado que se tratan de especificidades que, por tanto, escapan de la generalización, resultaría conveniente no introducirlas en la enseñanza de la morfología, aunque el profesor debe, obviamente, conocerlas. 
que también presenta bio-/-bio (biología-aerobio), por lo que estos dos elementos serían, sin lugar a dudas, temas cultos.

Como se ha señalado estos elementos cultos se asocian semántica y categorialmente a sustantivos o adjetivos. Sin embargo, ciertos elementos cultos, con el tiempo, acaban por desarrollar otros valores, como sucede en el caso de -dromo. Es decir, ¿este tema culto aporta la misma significación en hipódromo que en botellódromo? La respuesta a la pregunta es negativa y la explicación a esa respuesta es que en la evolución de este elemento culto ha intervenido un proceso de gramaticalización, como se va a mostrar a continuación.

La gramaticalización no es un fenómeno exclusivamente diacrónico, pero su incidencia se evidencia mejor si se adopta una perspectiva histórica. La gramaticalización consiste, a grandes rasgos, en la conversión de un elemento léxico en un elemento gramatical o bien cuando un elemento gramatical se hace más gramatical ${ }^{15}$. Sirva como ejemplo el paso del sustantivo mens, -tis del latín al sufijo formador de adverbios -mente: como se puede observar pasamos de un elemento léxico (un sustantivo en latín totalmente autónomo) a un elemento gramatical (un sufijo en español que carece, como todo afijo, de autonomía funcional).

La gramaticalización implica desde la perspectiva semántica una abstracción del significado ${ }^{16}$ como se desprende del ejemplo aportado, de modo que si se observa en la evolución de un determinado elemento un cambio de significación que consiste en su abstracción podemos considerar que se está gramaticalizando. Este hecho es lo que nos permite afirmar que el proceso de formación de palabras que generó la palabra hipódromo no es el mismo que el que dio lugar a sambódromo,

${ }^{15}$ No es el objetivo de este trabajo ahondar en los procesos de gramaticalización y de lexicalización a nivel teórico, sino mostrar sus posibilidades explicativas y su utilidad a la hora de delimitar determinados procesos de formación de palabras. Para un análisis de este proceso en el ámbito de la formación de palabras, véase Buenafuentes (2013).

${ }^{16}$ Esta abstracción consiste principalmente en que las unidades gramaticalizadas asumen valores cercanos a los que poseen los adverbios, las preposiciones y otro tipo de elementos que reciben el nombre de palabras gramaticales. 
aunque formalmente parezca que responden al mismo procedimiento. En la primera palabra compleja, el segundo elemento es un tema culto que posee el significado de 'carrera', pero en el segundo caso, -dromo ya no posee ese significado. Un sambódromo no es una 'carrera de sambas' sino que es un 'lugar en el que se baila samba'. Vemos, por consiguiente, que -dromo presenta en estas formaciones un valor locativo, semejante al que proporcionan los adverbios o preposiciones, lo que pone de manifiesto que ha abstraído su significado. Tal abstracción demuestra que este elemento en estas formaciones se comporta como un sufijo (dejaría, por tanto, de ser un tema) y que estas palabras serían resultado de un proceso de derivación no de composición culta (véase Buenafuentes, 2012).

Podemos observar el mismo proceso en el caso de -logía. El significado que aporta este tema culto en palabras como egiptología o mineralogía no es el mismo que en rumorología o sintomatología. En el primer caso, -logía es un tema culto con el significado de 'tratado, estudio, ciencia', mientras que en el segundo ha abstraído su significado hacia el de 'conjunto de'. Este valor claramente cercano al de los cuantificadores muestra que ha experimentado una gramaticalización que lo ha convertido en un sufijo. Por consiguiente, tanto -dromo como -logía poseerían dos realizaciones ${ }^{17}$ como formadores de palabras: una como temas cultos y otra con el valor gramaticalizado en el que actuarían como sufijos. Esta diferencia es claramente observable si se parte del significado para ir a la forma, sin entrar en aspectos de índole teórica que, aunque el profesor debe dominar, no es necesario que los alumnos conozcan. De todos modos, dada la complejidad de este aspecto sería conveniente reservar su enseñanza para Bachillerato.

El otro proceso de carácter diacrónico que resulta operativo para dar cuenta de la evolución que experimentan los procesos de formación de palabras es la lexicalización. Bajo esta denominación se esconden dos tipos de fenómenos que, si bien comparten alguna característica, no son totalmente identificables.

${ }_{17}$ De hecho, esa doble caracterización ya se lleva a cabo para el formante -oide por parte del DLE (véase RAE y ASALE, 2014). 
En primer lugar, la lexicalización puede hacer referencia a la pérdida de composicionalidad semántica, es decir, a que el significado de la forma compleja ya no se deduzca a partir de los elementos que la integran. Si no se introduce este concepto de lexicalización no se puede explicar que flequillo ${ }^{18}$ 'porción de cabello', sillón 'silla sin brazos' o camarote 'compartimento de un barco' no tengan estructura morfológica compleja ${ }^{19}$. A pesar de que en ellos se pueda reconocer la existencia de dos constituyentes (un sustantivo y un sufijo apreciativo), el significado de la formación no se deduce del sentido de estos dos elementos. Por consiguiente, flequillo, sillón y camarote son voces que han experimentado un proceso de lexicalización. De hecho, estos procesos de lexicalización se remontan incluso a la evolución del latín al español: voces como músculo o abeja son en realidad producto de un proceso de lexicalización, ya que sus respectivos étimos, musculum 'ratoncito' y apiculam 'abejita', estaban formados por el sufijo apreciativo diminutivo -culum /-culam y, con el paso del tiempo, aparte de experimentar los procesos fónicos regulares en el tránsito del latín al español, también pierden la composicionalidad, de modo que las dos voces pasan a ser palabras simples en las que ya no se reconoce su origen como derivados apreciativos. Otros ejemplos semejantes, pero que se desarrollan en el mismo español, son los casos de buhardilla y ardilla. Estas voces originariamente están constituidas por una base (buharda y arda, respectivamente) y el sufijo apreciativo -illa, pero ambas se lexicalizaron, de modo que se ha perdido su composicionalidad y se consideran dos palabras simples que, por consiguiente, carecen de una estructura morfológica compleja.

No se trata de que los estudiantes tengan que reconocer casos como los presentados, que se remonten al latín, pero el profesor puede usar este tipo de ejemplos para mostrar que la lengua está en continuo cambio y que lo que fue una formación compleja en el momento de su creación dejó de serlo con el paso del tiempo. De todos modos, en la mayoría de los casos, reconocer un proceso de lexicalización no resulta

\footnotetext{
${ }^{18}$ Algunas de estas voces pueden tener una doble lectura: una en la que se mantiene la composicionalidad y otra en la que se produce la lexicalización, como se puede comprobar en el ejemplo (7)

${ }^{19}$ Nos referimos a que no son resultado de un proceso de formación de palabras.
} 
complicado para un hablante nativo y puede ejercitarse fácilmente mediante pares mínimos como el siguiente:

a. Le ha cortado el flequillo [a María]

b. Le ha cortado el flequillo [a la cortina]

Como se desprende de este par mínimo, solo en la oración de b. el sustantivo derivado flequillo posee un significado composicional $\mathrm{y}$, por tanto, tiene una estructura morfológica compleja (fleco + illo = 'fleco pequeño'), mientras que, en la oración de a., flequillo ha experimentado un proceso de lexicalización, ya que su significado no es deducible porque refiere a una parte del pelo que cae sobre la frente. El mismo proceso de pérdida de composicionalidad semántica se puede observar en las siguientes oraciones:

a. Dibujó el diente de león

b. Dibujó el diente del león

Mientras que en la oración de b. la composicionalidad semántica es evidente (los significados son deducibles a partir de todos los elementos que integran la oración), en la oración de a., diente de león posee un significado opaco, que ha experimentado una lexicalización, porque su significado no se deriva de sus elementos constitutivos. Este tipo de expresiones pluriverbales lexicalizadas que poseen valor denominativo se conocen con el nombre de compuestos sintagmáticos. Cuando una construcción está lexicalizada (como es el caso de los compuestos sintagmáticos) funciona como un bloque a todos los efectos $\mathrm{y}$, por consiguiente, no puede ser modificada ni alterada desde el punto de vista interno (mediante la inserción de elementos o su sustitución). Como se observa en el par mínimo, la diferencia entre la oración de a. y la de b. desde el punto de vista formal es la presencia del artículo definido en b. Ese elemento es precisamente el que hace que la construcción se interprete literalmente (el diente del animal), mientras que en a. diente de león 'planta' sería una construcción lexicalizada y no puede ver alterada su estructura. Se considera, en este caso, que la incorporación del artículo desautomatiza el compuesto sintagmático (hace que la estructura adquiera composicionalidad). En definitiva, este concepto de lexicalización no resultaría difícil de introducir en niveles 
de aprendizaje iniciales a partir de contrastes como los expuestos hasta ahora, pues permiten partir del conocimiento que nuestros estudiantes tienen como hablantes y orientar la reflexión sobre la formación de las palabras desde el significado hasta la forma.

El otro sentido con que se emplea el término lexicalización hace referencia a la conversión de un elemento gramatical en un elemento léxico o cuando un elemento léxico se hace más léxico. En estos casos, el elemento lexicalizado adquiere autonomía funcional, de modo que se convierte en una palabra a todos los efectos. Este proceso de lexicalización tiene interés en el ámbito de la formación de palabras cuando los afijos o los temas (formas ligadas) que se utilizan en la lexicogénesis se lexicalizan y pasan a ser palabras autónomas (formas libres) que se utilizan, además, para formar nuevas palabras. Obsérvense los siguientes ejemplos:
a. cardiocirugía, cardiopatía, cardiovascular
b. ejercicios de cardio, hacer cardio
c. cardioamigos, cardiofunk

El tema culto cardio- 'corazón' interviene en la formación de compuestos cultos como los de a. Sin embargo, este tema culto, a partir del acortamiento de la palabra cardiovascular, ha sufrido un proceso de lexicalización, de modo que ha adquirido autonomía y un significado que ya no es el de 'corazón' sino el de 'cardiovascular, ejercicios cardiovasculares', como se observa en los ejemplos de b. en los que cardio ya se emplea como una palabra simple. Con este sentido, participa en la formación de nuevas palabras como en cardioamigos (amigos que comparten la ejercitación mediante ejercicios cardiovasculares) o cardiofunck (un estilo de baile que supone la ejercitación cardiovascular) ${ }^{20}$.

Este es el mismo proceso experimentado por el prefijo tele'lejos' a partir de televisión o por el tema culto bio- 'vida' a partir de biológico (véase Martín García, 2017a y 2017b). Ambas voces sufren un acortamiento en tele- y bio- con el significado de 'televisión' y

${ }^{20}$ Ejemplos extraídos del CORPES XXI de la Real Academia Española. 
'biológico', respectivamente, y, a partir de ahí, se lexicalizan (la tele, productos bio), de modo que pueden intervenir con ese nuevo valor en la formación de nuevos compuestos como telebasura, telenovela, biocombustible o bioagricultura. El proceso de lexicalización en estos casos resulta relevante morfológicamente porque, por ejemplo, televisión sería una palabra prefijada pero telebasura sería compuesta. En otros casos, como en cardio- o bio-, sería relevante porque tendríamos dos tipos de compuestos: en el caso de cardio- 'corazón' y bio- 'vida', el resultado sería un compuesto culto, mientras que cuando cardio- significa 'ejercicios cardiovasculares' y bio- 'biológico' nos moveríamos en la esfera de la composición léxica. La incidencia de los procesos de lexicalización, como se ha demostrado, resulta imprescindible para una adecuada caracterización de los procesos de formación de palabras.

A pesar de que cabría secuenciar, como se ha señalado, estos procesos en función del nivel de aprendizaje (lexicalización podría ser adecuado para niveles iniciales y gramaticalización para niveles superiores), introducir estos dos conceptos de marcado valor diacrónico en la enseñanza de la morfología ${ }^{21}$, como se ha mostrado, es operativo en dos aspectos fundamentales: en primer lugar, porque proporcionan una visión de la morfología más real y dinámica, contraria al estatismo con el que se suele mostrar esta parte de la gramática en Secundaria y en Bachillerato y, en segundo lugar, porque brindan a los estudiantes herramientas con las que decodificar y codificar palabras complejas, lo que, sin duda, contribuye a la mejora de su competencia lingüística.

\section{Conclusiones}

En este trabajo se ha demostrado la operatividad de determinados conceptos y procesos de índole diacrónica en la enseñanza de la morfología en Secundaria y en Bachillerato. A pesar de que se asume que el análisis de la estructura interna de la palabra y de las reglas de formación de palabras debe efectuarse desde la sincronía,

${ }_{21}$ Y también en la enseñanza de la gramática en general, tal y como pone de manifiesto el trabajo de Brandani y Bohrn (2019). 
el empleo de determinados conceptos diacrónicos, como el de alomorfía diacrónica, el de las restricciones etimológicas de las RFP o el de tema de palabra inexistente, permite explicar la estructura interna de muchas voces complejas del español que quedarían fuera del análisis morfológico si solo se tiene en cuenta el plano sincrónico. Por consiguiente, resultan ser conceptos operativos porque amplían el carácter explicativo y descriptivo del enfoque sincrónico. En este sentido, la introducción de aspectos diacrónicos en la enseñanza de la morfología en Secundaria y en Bachillerato, secuenciados según el nivel de aprendizaje, permite establecer conexiones con otras lenguas del currículum (el latín, por ejemplo), lo cual fomenta la transversalidad interlingüística.

También se ha podido comprobar cómo la incorporación de procesos que son principalmente (aunque no exclusivamente) diacrónicos, como la gramaticalización y la lexicalización, contribuye a una mejor delimitación de los mecanismos lexicogenésicos, favorece la codificación y decodificación de las palabras complejas y proporciona una visión dinámica de la morfología. Si bien el alumnado no necesita dominar estos dos procesos del cambio linguístico (y menos en estadios iniciales de aprendizaje), consideramos que el profesorado puede utilizarlos como mecanismo para la reflexión sobre los procesos de formación de palabras y de la huella que deja en ellos el paso del tiempo, ya que, en definitiva:

Un estado de lengua es solo un instante en una evolución infinita; el habla de hoy es hija y nieta del habla de ayer y de una serie de ayeres y de anteayeres que se alejan en el pasado; y la comprensión perfecta de lo que hoy es vivo precisa, no exclusivamente, pero sí también, de la luz que puede darnos el conocimiento de lo que era vivo ayer (Seco, 1987, p. 55).

La diacronía no debe ser el centro de la enseñanza de la morfología en Secundaria y en Bachillerato, pero conocer ciertos conceptos y procesos de índole histórica proporciona argumentos más sólidos y amplios para enseñar la morfología de manera reflexiva y competencial, de modo que su aprendizaje acabe siendo significativo. 


\section{Referencias bibliográficas}

Bravo Bosch, M. C. (1994). Introducción de la historia de la lengua en la clase de gramática. En J. Sánchez Lobato e I. Santos Gargallo (Eds.), Actas del IV Congreso Internacional de ASELE. Problemas y métodos en la enseñanza del español como lengua extranjera (pp. 391-397). Madrid: SGEL.

Bosque, I. (2017). Cómo nos ven. La percepción social de la gramática y de los gramáticos. Ms. Universidad Nacional de Rosario (Argentina).

Bosque, I., y Gallego, Á. (2016). La aplicación de la gramática en el aula. Recursos didácticos clásicos y modernos para la enseñanza de la gramática. Revista de lingüística teórica y aplicada, 54(2), 63-83. doi: 10.4067/S0718-48832016000200004.

Brandani, L., y Bohrn, A. (2019). Lexicalización y gramaticalización en la enseñanza de la variación lingüística. Quintú Quimün. Revista de lingüística, 3, 1-19.

Buenafuentes, C. (2012). Composición y relaciones espaciales: procesos de gramaticalización y lexicalización. En E. Bernal, C. Sinner y M. Emsel (Eds.), Tiempo y espacio en la formación de palabras (pp. 199-213). Munich: Peniope.

Buenafuentes, C. (2013). Sobre formación de palabras y los procesos de gramaticalización. En I. Pujol Payet (Ed.), Formación de palabras y diacronía (pp. 21-48). A Coruña: Servizo de Publicacións Universidade da Coruña (Anexos de la Revista de Lexicografía, 19).

Buenafuentes, C. (2020). Morfología y pares mínimos: algunos ejemplos para su implementación en secundaria. Revista de gramática orientada a las competencias, 3(1), 1-17. doi: 10.5565/rev/regroc.52.

Buenafuentes, C. (2021). Main compounding types in Spanish: Synchronic issues. En A. Fábregas, V. Acedo, G. Amstrong, M. Cuervo e I. Pujol (Eds.), The Routledge Handbook of Spanish Morphology (pp. 285-302). London: Routledge.

Buenafuentes, C., y Fernández Serrano, I. (en prensa). Las categorías gramaticales. En Á. Gallego y E. Gutiérrez (Eds.), La enseñanza de la gramática: teoría, metodología y competencias. Madrid: Visor. 
Díez del Corral, E. (2015). El aprendizaje y la enseñanza del español a través de su historia lingüística: puesta en práctica y valoración en una clase de estudiantes francófonos (nivel B2+/C1). Porta Linguarum, 23, 221-232. doi: 10.30827/Digibug.53768.

Echenique, M. ${ }^{a}$ T. (2011). La historia como recurso explicativo y apoyo codificador en la obra académica actual. Revista de filología española, XC(1), 159-170. doi: 10.3989/rfe.2011.v91.i1.

Felíu, E. (2009). Palabras con estructura interna. En E. de Miguel (Ed.), Panorama de la lexicología (pp. 51-81). Barcelona: Ariel.

Fernández Martín, P. (2019). Gramática histórica del español para francófonos universitarios de nivel $\mathrm{C} 1$ : en torno a una propuesta didáctica. Huarte de San Juan. Filología y didáctica de la lengua, 19, 75-107.

García Medall, J. L. (2019). La morfología derivativa del español. En E. Ridruejo (Ed.), Manual de lingüística española (pp. 402431). Berlín: De Gruyter.

Gil, I. (2019). El análisis morfológico en el aula. Perspectiva, contenidos, actividades. Revista de gramática orientada a las competencias, 2(1), 43-66. doi: 10.5565/rev/regroc. 42.

Iglesias, Y. (2018). Temas de palabras inexistentes. Diccionario de formantes y aplicación a la BDME (tesis doctoral). Universidad de Santiago de Compostela, Santiago de Compostela. Recuperada de https://minerva.usc.es/xmlui/handle/10347/18118.

Lleal, C. (2008). Enseñanza del español y diacronía. Estudios de lingüística del español de la Universidad de Alicante, 22, 151-168. doi: 10.14198/ELUA2008.22.

Martín García, J. (2017a). Formación de palabras y diccionario: los elementos compositivos por acortamiento. En I. Sariego López, J. Gutiérrez Cuadrado y C. Garriga (Eds.), El diccionario en la encrucijada: de la sintaxis y la cultura al desafío digital (pp. 863-877). Santander: Escuela Universitaria de Turismo de Altamira-Asociación Española de Lexicografía Hispánica.

Martín García, J. (2017b). Los límites de la prefijación. En J. Pena (Ed.), Procesos morfológicos. Zonas de interferencia (pp. 77104). Santiago de Compostela: Anexos de la revista Verba.

Martín García, J., y Varela, S. (2012). La relevancia de la diacronía para la teoría morfológica. En M. Campos, R. Mariño, J. I. 
Pérez Pascual y A. Rifón (Eds.), “Assí como es de suso dicho”: estudios de morfología y léxico en homenaje a Jesús Pena (pp. 325336). San Millán de la Cogolla: Cilengua.

Martín Zorraquino, M. A. (2000). El legado de aquellos maestros: la enseñanza de la gramática histórica desde el bachillerato. Archivo de filología aragonesa, 56, 63-78.

Michelena, L. (1963). Lenguas y protolenguas. Salamanca: Acta Salmanticensia.

Real Academia Española, y Asociación de Academias de la Lengua Española (2009). Nueva gramática de la lengua española. Madrid: Espasa.

Real Academia Española, y Asociación de Academias de la Lengua Española (2014). Diccionario de la lengua española (DLE). Madrid: Espasa.

Real Academia Española, y Asociación de Academias de la Lengua Española (2019). Glosario de términos gramaticales (GTG). Salamanca: Ediciones Universidad de Salamanca.

Real Academia Española, y Asociación de Academias de la Lengua Española. Corpus del español del siglo XXI (CORPES XXI). Disponible en https://www.rae.es/banco-de-datos/corpes-Xxi.

Seco, M. (1987). Estudios de lexicografía española. Madrid: Paraninfo.

Serrano-Dolader, D. (2012). Tensiones entre sincronía y diacronía en la descripción de la morfología léxica del español: el caso de la Nueva gramática de la lengua española. En M. Campos, R. Mariño, J. I. Pérez Pascual y A. Rifón (Eds.), "Assí como es de suso dicho”: estudios de morfología y léxico en homenaje a Jesús Pena (pp. 485-498). San Millán de la Cogolla: Cilengua. 